\title{
Structure, Bonding, and Photoaffinity Labeling Applications of Dialkyldiazirines
}

\section{Tianyi Zhang \\ Alison E. Ondrus* (10)}

Department of Chemistry, Division of Chemistry \& Chemical Engineering, California Institute of Technology, Pasadena, California, 91125, USA aondrus@caltech.edu
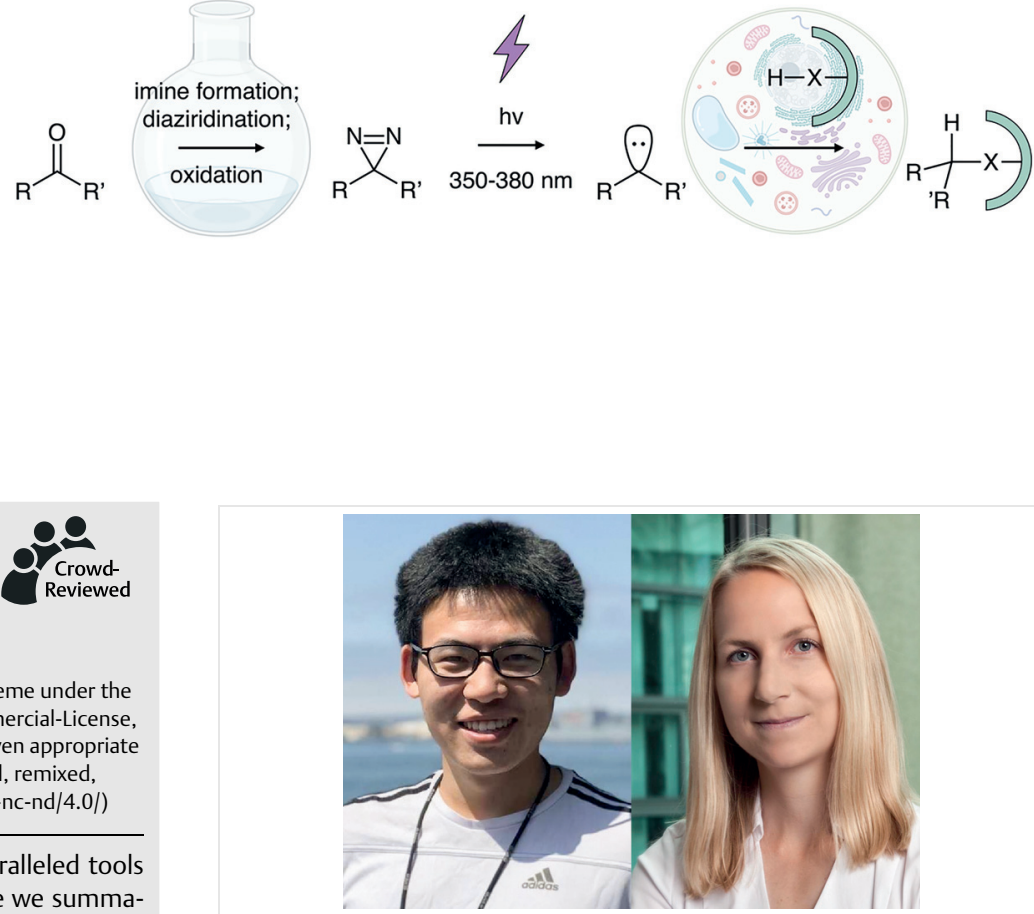

Abstract Dialkyldiazirine photoaffinity probes are unparalleled tools for the study of small molecule-protein interactions. Here we summarize the basic principles of structure, bonding, and photoreactivity of dialkyldiazirines, current methods for their synthesis, and their practical application in photoaffinity labeling experiments. We demonstrate the unique utility of dialkyldiazirine probes in the context of our recent photoaffinity crosslinking-mass spectrometry analysis to reveal a hidden cholesterol binding site in the Hedgehog morphogen proteins. Introduction

Structure, Bonding, and Spectral Properties

Photoreactivity

Synthesis

Application in Photoaffinity Labeling

Discovery of a Cholesterol-Hedgehog Protein Interface Conclusions and Outlook

Key words diazirine, carbene, photoreactivity, chemical biology, pro tein-ligand interactions

\section{Introduction}

Protein-ligand interactions are essential to the survival, development, and growth of all living organisms, and lie at the heart of modern drug development. Study of these interactions is crucial to the understanding of biological processes at the molecular and atomic levels. The ability of small molecule photoaffinity labeling probes to capture noncovalently bound proteins in a spatially precise manner makes them powerful tools for studying protein-ligand interactions. ${ }^{1,2}$ The diazirine group satisfies many of the criteria for an ideal photoactive unit: it is small, nonpolar, and
Tianyi Zhang was born in Jiangsu, China and is currently pursuing his BSC at the California Institute of Technology (USA) as a student researcher in the field of organic chemistry.

Alison E. Ondrus was born in Edmonton, Canada. After completing her BSc studies at the University of Alberta (Canada), her PhD at MIT (USA), and postdoctoral studies at Stanford University (USA), Alison assumed her position as an Assistant Professor at the California Institute of Technology (USA).

generally robust toward storage. Moreover, diazirines are inert in biological environments, absorb light at biocompatible wavelengths, and show diminished nonspecific binding upon photoactivation relative to other labeling probes. Recent years have seen an increase in the use of diazirines as probes to investigate protein-receptor interactions in vitro, in cells, and in whole organisms. ${ }^{3-5}$ To this end, two general classes of diazirine have been employed, namely (trifluoroalkyl)aryldiazirines ${ }^{6}$ and dialkyldiazirines. ${ }^{7}$ While the former have been widely used and are accessible by well-established methods, there are notable gaps in the study and synthesis of dialkyldiazirines. ${ }^{8}$ In this review, we focus on the bonding and structure, photochemical reactivity, and main synthetic routes to dialkyldiazirines, as well as their practical application in photoaffinity crosslinking experiments. As an example, we present our use of a diazirinyl cholesterol photoaffinity probe to identify an elusive bind- 
ing site in the Hedgehog morphogen proteins, 9,10 and provide a perspective on future opportunities in diazirinebased probe synthesis and application.

\section{Structure, Bonding, and Spectral Properties}

Diazirines are three-membered nitrogen-containing heterocycles that are constitutionally isomeric to the diazo group. ${ }^{11} 3 \mathrm{H}$-diazirine, the simplest member of the diazirine family, was first synthesized in $1961,{ }^{12}$ and its structure was later elucidated by rotational spectroscopy (Figure 1a). ${ }^{13}$ The $d_{\mathrm{NN}}$ and $d_{\mathrm{CN}}$ bond lengths in this molecule were initially characterized to be $1.228(3) \AA$ and $1.482(3) \AA$, respectively, with a R-C-R angle of $a_{\mathrm{HCH}}=$ $117(2)^{\circ}$. More recently, a combination of micro- and millimeter-wave rotational spectroscopies have provided the structure of $3 \mathrm{H}$-diazirines with greater precision, at values of $d_{\mathrm{NN}}=1.2280(25) \AA, d_{\mathrm{CN}}=1.4813(24) \AA$, and $a_{\mathrm{HCH}}=$ $120.54(27)^{\circ}$ (Figure 1a). ${ }^{14}$ Alongside these measurements, computational studies at $\operatorname{CCSD}(\mathrm{T})$ and MP2 levels of theory and with a mixed cc-pwCVQZ basis set have provided an equilibrium geometry in which $d_{\mathrm{NN}}=1.226 \AA$, $d_{\mathrm{CN}}=1.475$ $\AA$, and $a_{\mathrm{RCR}}=119.9^{\circ} \cdot{ }^{15}$ a)

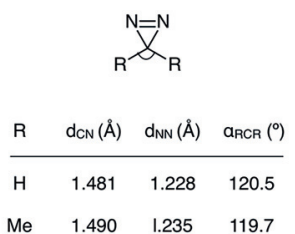

c)

\begin{tabular}{rl} 
property & typical range \\
\hline$\lambda_{1 \mathrm{R}} \mathrm{N}=\mathrm{N}$ & $1560-1585 \mathrm{~cm}^{-1}$ \\
$\mathrm{~N}=\mathrm{N}_{\mathrm{abs}}$ & $350-380 \mathrm{~nm}$ \\
$\mathrm{~N}=\mathrm{N}_{\mathrm{em}}$ & $380-410 \mathrm{~nm}$ \\
$\delta^{13} \mathrm{C} \mathrm{C}-\mathrm{N}_{2}$ & $\delta 20-30 \mathrm{ppm}$
\end{tabular}

b)

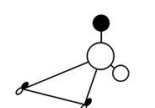

LUMO +1

LUMO<smiles></smiles><smiles>CC(C)C1CC1</smiles>

HOMO

HOMO -1

Figure 1 Structure, bonding, and spectroscopic properties of dialkyldiazirines. (a) Experimental bond lengths and angles for $3 \mathrm{H}$-diazirine and 3,3-dimethyldiazirine from refs. 15 and 16, respectively. (b) Frontier molecular orbitals of $3 \mathrm{H}$-diazirine, reproduced with permission from ref. 34. (c) Approximate ranges for dialkyldiazirine spectroscopic properties.

The corresponding parameters in the simplest dialkyldiazirine, 3,3-dimethyldiazirine, have been determined by rotational spectroscopy to be $1.228(3) \AA$ and $1.482(3) \AA$, respectively, with a R-C-R angle of $a_{\mathrm{HCH}}=117(2)^{\circ} \cdot{ }^{16}$ For reference, (trifluoromethyl)aryldiazirines have similar $d_{\mathrm{NN}}$ but slightly longer (by ca. $0.020 \AA$ ) $d_{\mathrm{CN}}$ bond lengths. ${ }^{17}$

Investigation of the ground-state electronic structure of 3H-diazirine in 1969 revealed the resemblance of its lowlying bonding molecular orbitals (MOs) to those in cyclopropane and cyclopropene. ${ }^{18}$ Namely, the low-lying MOs of diazirines consist largely of $\mathrm{C}_{2 \mathrm{~s}}$ atomic orbitals that form the

$\sigma$-bonding framework. However, the frontier orbitals of diazirines differ significantly from those of cyclopropane and cyclopropene, where the nitrogen lone pairs contribute to $\sigma$-bridged $\pi$-bonding within the three-membered ring. ${ }^{19,20}$ The highest occupied MO (HOMO), corresponding to the lone pairs on the nitrogen atoms, is an overall nonbonding Walsh-type MO with partial $\mathrm{C}-\mathrm{N} \pi$-bonding and $\mathrm{N}=\mathrm{N} \pi$-antibonding character (Figure 1b). The next lower-energy occupied orbital (HOMO-1) is a $\mathrm{N}=\mathrm{N} \pi$-bonding MO. The lowest unoccupied MO (LUMO) is a $\mathrm{N}=\mathrm{N} \pi^{*}$ orbital, while the next higher-energy virtual $\mathrm{MO}(\mathrm{LUMO}+1)$ is a $\mathrm{C}-\mathrm{H} \sigma^{*} \mathrm{MO}$ largely localized on the hydrogen atoms of the alkyl substituents. ${ }^{15}$ This portrait of the diazirine frontier MOs is supported by the observation that the $\mathrm{N}=\mathrm{N}$ stretching frequency in the first diazirine excited state decreases relative to the ground state, consistent with an $n \rightarrow \pi^{*}$ transition that weakens the $\mathrm{N}=\mathrm{N}$ bond.

The electronic absorption spectrum of $3 H$-diazirine was reported as early as in 1962, featuring a maximum absorption around $320 \mathrm{~nm}$ (Figure 1c). ${ }^{21,22}$ Later studies with 3,3dimethyldiazirine revealed maximum absorption values of 345 and $363 \mathrm{~nm}$ (ambient temperature, pentane), ${ }^{23}$ which fall in the general range of 350-380 nm for alkyl diazirines with substituents of various electronic and steric properties. ${ }^{24,25}$ The fluorescence spectra of dialkyldiazirines show a weak emission peak between 380 and $410 \mathrm{~nm} .{ }^{26}$ In the infrared (IR) region, 3H-diazirine features a characteristic $\mathrm{N}=\mathrm{N}$ stretch at $1560-1630 \mathrm{~cm}^{-1}$ of medium to strong intensity, consistent with typical values of $1560-1585 \mathrm{~cm}^{-1}$ for a variety of diazirine structures. ${ }^{25}{ }^{1} \mathrm{H}$ nuclear magnetic resonance (NMR) spectra of dialkyldiazirines can be distinguished by an upfield chemical shift of $\alpha$-diazirine protons by approximately $\delta 0.60-0.68 \mathrm{ppm}$ due to the anisotropic shielding effect of the diazirine $\pi$-system. ${ }^{26}$ For the same reason, the ${ }^{13} \mathrm{C}$ NMR chemical shift of the diazirine carbon in dialkyldiazirines generally falls in the range of $\delta 20-30$ ppm. A rich history of rotational spectroscopy characterization of diazirines provided the first clues about their structure; the interested reader is directed to detailed reviews on these topics. ${ }^{27}$

\section{Photoreactivity}

While initial investigations profiled the thermal reactivity of diazirines, computation of their excited-state electronic structure in 1966 explained their photochemical reactivity toward carbene formation. ${ }^{28-30}$ Subsequent experimental and theoretical studies have demonstrated that $n \rightarrow$ $\pi^{*}$ photoexcitation of an electron in the ground state $\left(\mathrm{S}_{0}\right)$ produces a singlet excited state $\left(S_{1}\right)$, which can follow a number of reaction pathways (Figure 2). ${ }^{31-36}$ First, the $S_{1}$ state can undergo simple fluorescence emission and return to the ground state $\left(\mathrm{S}_{0}\right)$. Second, photolysis can lead to extrusion of $\mathrm{N}_{2}$ and generation of a carbene in a doubly excit- 
ed singlet state with the $(\sigma)^{0}(\pi)^{2}$ configuration. In contrast, the thermolysis of diazirines typically yields a ground-state singlet carbene in the $(\sigma)^{2}(\pi)^{0}$ configuration. ${ }^{24,37}$ The singlet carbene generated by photolysis is responsible for concerted insertion into a nearby $\mathrm{R}-\mathrm{H}$ bond, which is the working principle for photoaffinity labeling.

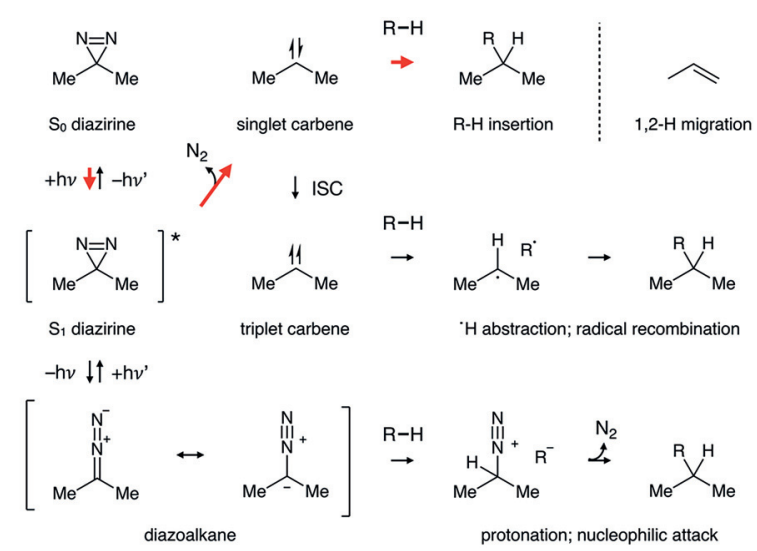

Figure 2 Photochemical reactivity of dialkyldiazirines. 3,3-Dimethyldiazirine is shown as an example. Red arrows indicate the desired insertion reaction for photoaffinity labeling.

Photoexcited diazirines are also susceptible to a number of nonproductive decay mechanisms that reduce the efficiency of photoaffinity labeling (Figure 2). A significant side-reaction of dialkyldiazirines with $\alpha$-protons is their high propensity to undergo a 1,2-hydride shift and expel $\mathrm{N}_{2}$ to form an olefin upon photolysis. ${ }^{38-40}$ While it was first proposed that the $S_{1}$ excited state undergoes a 1,2-hydride migration with concomitant loss of $\mathrm{N}_{2}$, more recent studies indicate that the hydride shift occurs after the generation of a singlet carbene from the $S_{1}$ state with an activation barrier of $2.6 \mathrm{kcal} \mathrm{mol}^{-1}$. The stepwise mechanism has been corroborated by kinetic isotope effect studies and high-level $a b$ initio computations on 3,3-dimethyldiazirine. ${ }^{41}$ Laser flash photolysis studies on $\alpha$-halodiazirines have demonstrated that the 1,2-hydride shift is accelerated by solvent polarity and by the branching of $\alpha$-alkyl substituents. ${ }^{42}$

A second predominant side-reaction of photoexcited diazirines is their facile rearrangement to diazo isomers upon exposure to $>340 \mathrm{~nm}$ light (Figure 2) ${ }^{43-50}$ While the diazo isomer can also yield an excited-state singlet carbene upon irradiation with $<315 \mathrm{~nm}$ light, ${ }^{51}$ emission at this wavelength is minimal from UV-A bulbs used in biological experiments. Unlike $\alpha$-aryl and $\alpha$-heteroatom-stabilized diazo compounds, dialkyldiazo compounds are prone to protonation at the diazo substituted carbon; displacement of $\mathrm{N}_{2}$ by a nucleophile can result in 'pseudo-carbene' photolabeling. ${ }^{49,52,53}$ Because the intermediate diazo compound has a lifetime of milliseconds, ${ }^{50}$ as compared to the picoseconds lifetime of the excited state carbene, this results in a broader labeling radius that is not diffusion limited.
While ${ }^{1} \mathrm{~S} \rightarrow{ }^{1} \mathrm{~T}$ intersystem crossing has been detected for dimethylcarbene in the gas phase, it has yet to be observed by laser flash photolysis at ambient temperature in nonpolar aprotic solvents. ${ }^{54,55}$ However, $\mathrm{O}_{2}$ trapping studies have indicated that intersystem crossing of the singlet carbene to the triplet is capable of kinetically competing with both inter- and intramolecular $\mathrm{R}-\mathrm{H}$ insertion reactions by singlet dimethylcarbene (Figure 2). ${ }^{23,56}$ Theoretical studies indicate that the singlet-triplet energy gap becomes significantly lower with increasing alkyl substitution $\alpha$ to the carbene. ${ }^{57-59}$ Triplet dimethylcarbenes can exhibit alternative forms of radical reactivity such as nonspecific atom abstraction and elimination. ${ }^{23}$

Excited-state carbenes that do not react by these pathways can also be quenched by insertion reactions with solvent. $\mathrm{O}-\mathrm{H}$ insertion by stabilized carbenes in protic solvent is well-documented, and facile insertion into solvent $\mathrm{O}-\mathrm{H}$ bonds has also been observed in less stabilized dialkylcarbenes based on alcohol trapping studies. ${ }^{23}$ For (trifluoromethyl)phenyldiazirine and analogues, a half-life of 160200 ns has been reported in polar aprotic solvents (e.g., pyridine, acetonitrile) and 150 ns in nonpolar solvents (e.g., hexafluorobenzene). ${ }^{60}$ The yield of $\mathrm{O}-\mathrm{H}$ insertion is highly influenced by solvent hydrogen bonding, where stronger hydrogen bonds reduce the extent of $\mathrm{O}-\mathrm{H}$ insertion. ${ }^{61}$ The various fates of photoexcited diazirines reduce crosslinking efficiencies, motivating further study of their behavior in biological systems and new chemical tactics to tune their reactivity.

\section{Synthesis}

The synthesis of dialkyldiazirines from carbonyl compounds was first published in $1960,{ }^{11}$ and subsequent protocols have followed this method relatively closely. ${ }^{8}$ Dialkyl diazirine formation from a carbonyl substrate typically proceeds in three steps (Table 1): (1) Condensation of the carbonyl 1 with a nucleophilic nitrogen atom source to form an imine; (2) Addition of an electrophilic nitrogen atom source across the $\mathrm{C}=\mathrm{N}$ double bond of the imine to form a diaziridine 2 ; (3) Oxidative dehydrogenation of the diaziridine to a diazirine 3.

Table 1 provides examples of reagents and conditions that are commonly used for the synthesis of dialkyldiazirines. ${ }^{12,62-75}$ Formation of the imine from $\mathbf{1}$ is typically carried out using ammonia, either in methanolic solution (entries 5-11) or condensed as a liquid (entries 1-4). For sterically hindered carbonyl substrates, imines can be accessed in two steps via formation of the cyclohexylamine imine/enamine or the $O$-nitroso oxime before treatment with ammonia (entries 10 and 11). For diaziridination, hydroxylamine- $O$-sulfonic acid (HOSA, or HAOSA) is the dominant source of the second nitrogen atom. ${ }^{76} \mathrm{~A}$ variety of oxidants have been reported for dehydrogenation of the dia- 
Table 1 Summary of Reagents and Conditions for Synthesis of Dialkyldiazirines

\begin{tabular}{|c|c|c|c|c|}
\hline Entry & Conditions 'a' & Conditions 'b' & Yield (\%) & Reference \\
\hline $1^{\mathrm{a}}$ & $\mathrm{NH}_{3}(I), \mathrm{HOSA}^{\mathrm{b}}$ & $\mathrm{KOH}$, air & $44-90$ & 62 \\
\hline 2 & $\mathrm{NH}_{3}(\mathrm{l}), \mathrm{HOSA}$ & $t$-BuOK, air & 62 & 63 \\
\hline 3 & $\mathrm{NH}_{3}(\mathrm{l}), \mathrm{HOSA}$ & $\mathrm{I}_{2}, \mathrm{Et}_{3} \mathrm{~N}$ & $22-57$ & 64-70 \\
\hline 4 & $\mathrm{NH}_{3}(\mathrm{l}), \mathrm{HOSA}$ & $\mathrm{Ag}_{2} \mathrm{O}$ & $27-43$ & 64 \\
\hline 5 & $\mathrm{NH}_{3}(\mathrm{MeOH}), \mathrm{HOSA}$ & $\mathrm{I}_{2}, \mathrm{Et}_{3} \mathrm{~N}$ & $29-70$ & 71,72 \\
\hline 6 & $\mathrm{NH}_{3}(\mathrm{MeOH}), \mathrm{HOSA}$ & $\mathrm{Ag}_{2} \mathrm{O}$ & $14-27$ & 12,73 \\
\hline 7 & $\mathrm{NH}_{3}(\mathrm{MeOH}), \mathrm{HOSA}$ & $\mathrm{CrO}_{3}$ & $48-50$ & 74 \\
\hline 8 & $\mathrm{NH}_{3}(\mathrm{MeOH}), \mathrm{tBuOCl}$ & $\mathrm{AgNO}_{3}, \mathrm{NaOH}$ & 27 & 12 \\
\hline 9 & $\mathrm{NH}_{3}(\mathrm{MeOH}), \mathrm{HOSA}$ & $\mathrm{AgNO}_{3}, \mathrm{NaOH}$ & 25 & 12 \\
\hline 10 & $\begin{array}{l}\text { 1. } \mathrm{CyNH}_{2}, \mathrm{TFA} \\
\text { 2. } \mathrm{NH}_{3}(\mathrm{MeOH}), \mathrm{HOSA}\end{array}$ & $\mathrm{I}_{2}, \mathrm{Et}_{3} \mathrm{~N}$ & 58 & 75 \\
\hline 11 & $\begin{array}{l}\text { 1. } \mathrm{NH}_{2} \mathrm{OH} \\
\text { 2. } \mathrm{NaNO}_{2}, \mathrm{AcOH} \\
\text { 3. } \mathrm{NH}_{3}(\mathrm{MeOH}) \\
\text { 4. } \mathrm{HOSA}\end{array}$ & $\mathrm{I}_{2}, \mathrm{Et}_{3} \mathrm{~N}$ & 29 & 72 \\
\hline
\end{tabular}

a One-pot synthesis

${ }^{b}$ HOSA: hydroxylamine-O-sulfonic acid

ziridine to the diazirine, including $\mathrm{Ag}_{2} \mathrm{O}$ (entries 4 and 6), $\mathrm{AgNO}_{3}$ (entries 8 and 9), and $\mathrm{CrO}_{3}$ (entry 7). Recently, onepot synthesis of diazirines from dialkyl ketones in air under strongly basic conditions in liquid ammonia has been reported to provide yields of up to $90 \%$ (entry 1 ). Notably, a standard protocol for the synthesis of (trifluoromethyl)phenyldiazirines - namely, formation of an 0 -tosyl oxime, diaziridination using $\mathrm{NH}_{3}$, and oxidation - is unsuitable for the synthesis of dialkyldiazirines, likely due to undesired Beckmann rearrangement of the $O$-tosyl oxime intermediate.

The typical yields for dialkyldiazirines using the protocols listed in Table 1 are generally less than 50\%, with a few exceptions noted above. The harsh conditions and relatively poor yields for diazirine introduction present a bottleneck in the synthesis of many dialkyldiazirine probes. As a result, diazirine-containing fragments are often incorporated into more complex probe structures at a late stage of synthesis. While improving overall yields, this strategy can significantly modify the structure of the parent molecule and limit the location of the reactive carbene, motivating the development of new preparative methods.

\section{Application in Photoaffinity Labeling}

Diazirine-containing molecules can serve as biochemical tools to elucidate noncovalent small molecule binding sites in proteins. While the crosslinking radius for dialkyldiazirine labeling upon photoactivation has yet to be rigorously defined, present studies report an experimental value of approximately $9 \AA .{ }^{77}$ Depending on substitution, the lifetime of a carbene generated from a diazirine in nonpolar solvents can be on the scale of nanoseconds to microseconds, but is significantly shorter (diffusion-controlled, <1 ns) upon the addition of polar solvents like amines and water. The shorter lifetime of dialkyldiazirines in aqueous environment theoretically imparts a shorter radius and a better resolution for photoaffinity labeling. Due to fractional quantum yields and various sources of interference in biological samples, exposure times for protein and cell photolabeling experiments are generally on the scale of minutes to hours.

A typical protocol for protein photoaffinity labeling with a diazirine probe entails incubation of the probe with the sample in vitro, in cell lysates, or in live cells. ${ }^{78}$ Subsequently, samples are irradiated with UV-A light, commonly using $365 \mathrm{~nm}$ bulbs, for 1 to 60 minutes while the sample is incubated at low to room temperature $\left(0-23^{\circ} \mathrm{C}\right)$. The desired dialkylcarbene species generated by photoactivation of the diazirine can covalently attach to amino acids within the labeling radius in a residue-agnostic manner. This serves as an excellent alternative to immunolabeling methods, especially for proteins of unknown identity or proteins that lack suitable antibodies.

\section{Discovery of a Cholesterol-Hedgehog Pro- tein Interface}

The Hedgehog (Hh) morphogen proteins play critical roles in the embryonic development of humans and higher organisms. ${ }^{9,10,79}$ Hh proteins are initially translated as 35$50 \mathrm{kDa}$ proteins that consist of an N-terminal 'Hedge' (signaling) domain and a C-terminal 'Hog' (catalytic) domain. To form the active morphogen, the Hog domain cleaves the protein backbone and simultaneously modifies the terminal carboxy group of the Hedge domain as a cholesterol ester. ${ }^{80}$ While the mechanism of the autoproteolysis step is well-established, the noncovalent interactions that facilitate cholesterol esterification remain obscure. To define the noncovalent cholesterol binding site within the Hog domain, we employed a diazirine analogue of cholesterol as a photoaffinity labeling probe. ${ }^{10}$

We first evaluated the properties of a nonsymmetrical dialkyldiazirine representative of our photocholesterol probe (Figure 3 ). While structure and bonding of symmetrical dialkyldiazirines have been extensively analyzed, the features of nonsymmetrical dialkyldiazirines are not widely 
reported. In particular, schematic representations of the MOs of a nonsymmetrically substituted dialkyldiazirine have yet to be reported. We therefore computationally evaluated the MOs of 3-ethyl-3-methyldiazirine, the simplest non-symmetric dialkyldiazirine, at the B3LYP/6-311G(d,p) level of theory. ${ }^{81-85}$ Our MOs for 3-ethyl-3-methyldiazirine closely resemble those in 3,3-dimethyldiazirine, ${ }^{20}$ suggesting that the substitution by different straight-chain alkyl groups in methyl diazirines will have a minor effect on their frontier MOs. Calculation of the equilibrium geometry using Gaussian 16 at the B3LYP/6-311+G(d,p) level of theory yielded $d_{\mathrm{NN}}=1.2285 \AA d_{\mathrm{CN}}=1.4795 \AA$, and $\alpha_{\mathrm{RCR}}=117.49^{\circ}$, which is in decent agreement with X-ray crystal structures of glycosylidene-derived diazirines ${ }^{86}$ and substituted aziadamantanes. ${ }^{87,88}$ Our calculated $\mathrm{N}=\mathrm{N}$ stretching frequency of $1703 \mathrm{~cm}^{-1}$ for 3-ethyl-3-methyldiazirine corresponds well with the empirical $\mathrm{N}=\mathrm{N}$ stretching frequency of other dialkyldiazirines. ${ }^{24}$ a)

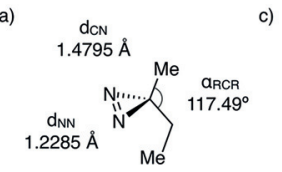

b)

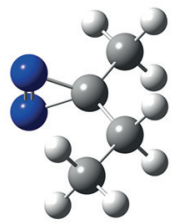

c)

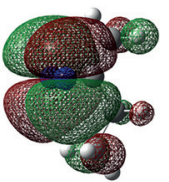

LUMO

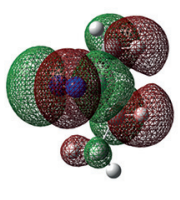

HOMO

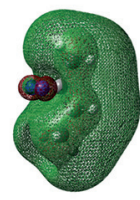

LUMO +1

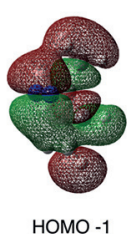

Figure 3 Geometry and frontier molecular orbitals of 3-methyl,3-ethyldiazirine calculated at the B3LYP/6-311G(d,p) level of theory. For comparison, a schematic representation of the frontier molecular orbitals of dimethyldiazirine is shown in Figure 1b.

Owing to the poor solubility of 27-nor-25-ketocholesterol (4) in polar solvents, conversion to 25-diazirinylcholesterol 5 ('photocholesterol') under conventional conditions afforded rather low yields (<20\%) (Figure 4a). ${ }^{10}$ Gratifyingly, two-step imine formation with cyclohexylamine and TFA followed by $\mathrm{NH}_{3}$ in $\mathrm{MeOH}$, diaziridination with $\mathrm{HO}-$ $\mathrm{SA}$, and oxidation with $\mathrm{I}_{2}$ gave photocholesterol $\mathbf{5}$ in a substantially higher yield (61\%). ${ }^{71}$ We assessed photoactivation of 5 as a $10 \mathrm{mM}$ solution in $N, N$-dimethylformamide (DMF) using a five-tube $368 \mathrm{~nm}$ UV lamp. The $\mathrm{N}=\mathrm{N}$ absorbance at $350 \mathrm{~nm}$ was observed to follow approximately first-order decrease with a half-life of around 2 minutes (Figure 4c).

To identify the noncovalent binding site for cholesterol in the Hog domain of the Hh protein, we incubated photoaffinity labeling probe $\mathbf{5}$ with active, purified Hog protein at low temperature $\left(0^{\circ} \mathrm{C}\right)$ to trap the noncovalent 5:Hog complex (Figure 4b). Photocrosslinking, followed by SDS-
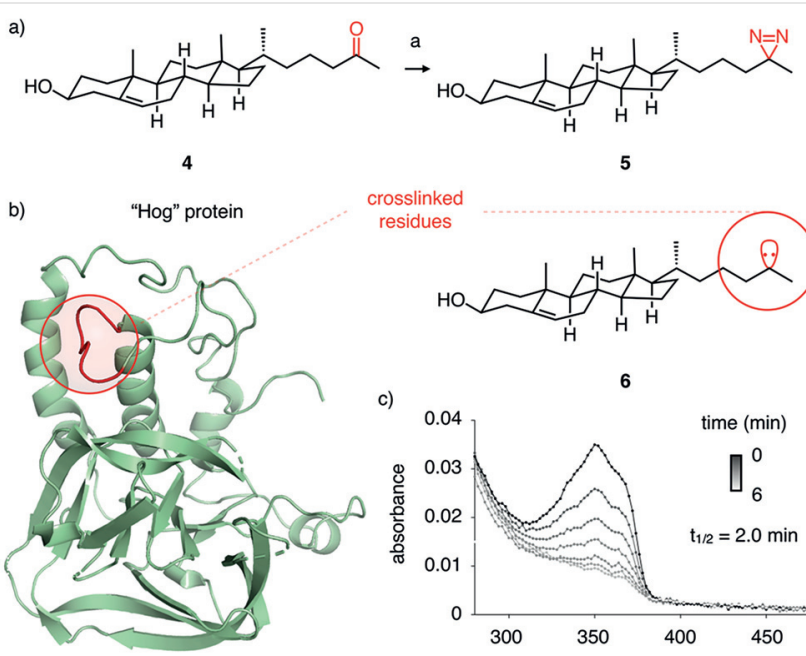

$$
\begin{aligned}
& \text { rosslinked } \\
& \text { residues }
\end{aligned}
$$
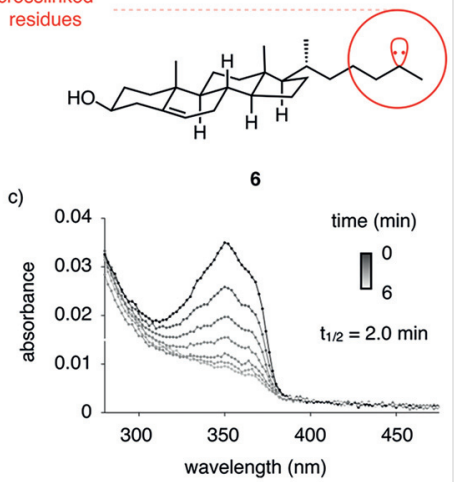

Figure 4 Elucidation of the cholesterol binding site in the Hedgehog protein using a photocholesterol probe. (a) Synthesis of photocholesterol 5. Conditions: (a) (i) $\mathrm{CF}_{3} \mathrm{CO}_{2} \mathrm{H}, \mathrm{CyNH}_{2}, 17 \mathrm{~h}, 145^{\circ} \mathrm{C}$; (ii) $\mathrm{NH}_{3}$, $\mathrm{MeOH}, 4 \mathrm{~h}, 0^{\circ} \mathrm{C}$; (iii) HOSA, $24 \mathrm{~h}, 0-23^{\circ} \mathrm{C}$; (iv) $\mathrm{I}_{2}$, $\mathrm{Et}_{3} \mathrm{~N}, \mathrm{CH}_{2} \mathrm{Cl}_{2}, 1 \mathrm{~h}, 0-23$ ${ }^{\circ} \mathrm{C}$ (61\%: overall yield). (b) Model of the apo protein, showing the peptide crosslinked by $\mathbf{5}$ upon photolysis. (c) Fluorescence absorbance spectrum of 5 upon continuous irradiation with $368 \mathrm{~nm}$ light.

PAGE separation, in-gel digestion, and mass spectrometry analysis revealed a predominant peptide that contained the mass of the 5-derived modification. Crosslinking occurred at either a histidine, a leucine, or a glutamate residue in this sequence, consistent with trapping of a highly reactive 5derived carbene and/or its diazo isomer. Localization of the modified peptide provided a pivotal constraint for molecular dynamics analysis of the Hog-cholesterol binding site, revealing a dynamic, hydrophobic environment that coordinates esterification.

\section{Conclusions and Outlook}

This review has summarized the bonding and structure, photoreactivity, synthesis, and biochemical application of dialkyldiazirines as photoaffinity labeling probes. The use of diazirines for photoaffinity labeling will expand in years to come, and significant opportunities remain to improve their synthesis and utility. Design of $\alpha$-substituted diazirines that favor singlet R-H insertion may avoid facile alkene formation and/or spatially imprecise diazo trapping. Preparative methods that capitalize on recent advances in nitrogen atom insertion chemistry using transition-metalbased atom-transfer reagents may enable milder, higheryielding diazirine synthesis for late-stage installation. ${ }^{89}$ Moving forward, a better understanding of the chemical properties of these dialkyldiazirine-based probes in biological systems - in particular, their labeling radius, lifetimes, 
and quenching behavior - will also be required to fine-tune the reactivity and strategically insert the dialkyldiazirine moiety within probe molecules of interest.

\section{Conflict of Interest}

The authors declare no conflict of interest.

\section{Funding Information}

Support was provided by Caltech start-up funds for A.E.O. and a John Stauffer Charitable Trust SURF Fellowship for T.Z.

\section{Acknowledgment}

We thank Dr. Y.-S. Cheng and Prof. R. G. Hadt for invaluable input during the preparation of this manuscript.

\section{References}

(1) Bayley, H.; Knowles, J. R. Methods Enzymol. 1977, 46, 69.

(2) Chowdhry, V.; Westheimer, F. H. Annu. Rev. Biochem. 1979, 48, 293.

(3) Halloran, M. W.; Lumb, J. Chem. Eur. J. 2019, 25, 4885.

(4) Dubinsky, L.; Krom, B. P.; Meijler, M. M. Bioorg. Med. Chem. 2012, 20, 554 .

(5) Blencowe, A.; Hayes, W. Soft Matter 2005, 1, 178.

(6) Brunner, J.; Senn, H.; Richards, F. M. J. Biol. Chem. 1980, 255, 3313.

(7) Das, J. Chem. Rev. 2011, 111, 4405.

(8) Hill, J. R.; Robertson, A. A. B. J. Med. Chem. 2018, 16, 6945.

(9) Purohit, R.; Peng, D. S.; Vielmas, E.; Ondrus, A. E. Commun. Biol. 2020, 3, 250

(10) Mafi, A.; Purohit, R.; Vielmas, E.; Lauinger, A. R.; Lam, B.; Cheng, Y.-S.; Zhang, T.; Huang, Y.; Kim, S.-K.; Goddard, W. A.; Ondrus, A. E. PLOS One 2021, e0246814.

(11) Paulsen, S. R. Angew. Chem. 1960, 72, 781.

(12) Schmitz, E.; Ohme, R. Chem. Ber. 1961, 94, 2166.

(13) Pierce, L.; Dobyns, S. V.J. Am. Chem. Soc. 1962, 84, 2651.

(14) Verma, U. P.; Möller, K.; Vogt, J.; Winnewisser, M.; Christiansen, J. J. Can. J. Phys. 1985, 63, 1173.

(15) Puzzarini, C.; Gambi, A.; Cazzoli, G. J. Mol. Struct. 2004, 695, 203.

(16) Wollrab, J. E.; Scharpen, L. H.; Ames, D. P.; Merritt, J. A. J. Chem. Phys. 1968, 49, 2405.

(17) Barton, D. H. R.; Jaszberenyi, J. C.; Theodorakis, E. A.; Reibenspies, J. H. J. Am. Chem. Soc. 1993, 115, 8050.

(18) Kochanski, E.; Lehn, J. M. Theor. Chim. Acta 1969, 14, 281

(19) Liang, C.; Allen, L. C. J. Am. Chem. Soc. 1991, 113, 1878.

(20) Han, M. S.; Cho, H.-G.; Cheong, B.-S. Bull. Korean Chem. Soc 1999, 20, 1281.

(21) Merritt, J. A. Can. J. Phys. 1962, 40, 1683.

(22) Robertson, L. C.; Merritt, J. A. J. Chem. Phys. 1972, 56, 2919.

(23) Modarelli, D. A.; Morgan, S.; Platz, M. S. J. Am. Chem. Soc. 1992, 114,7034

(24) Liu, M. T. H. Chem. Soc. Rev. 1982, 11, 127.

(25) Yamamoto, N.; Bernardi, F.; Bottoni, A.; Olivucci, M.; Robb, M. A.; Wilsey, S. J. Am. Chem. Soc. 1994, 116, 2064.
(26) Heine, H. W.; Zimmer, R. In Chemistry of Heterocyclic Compounds, Vol. 42(2); Hassner, A., Ed.; John Wiley \& Sons: Weinheim, 1983, 547.

(27) Winnewisser, M.; Möller, K.; Gambi, A. In Chemistry of Diazirines, Vol. 1; Liu, M. T., Ed.; CRC Press: Boca Raton, 1987, 19.

(28) Frey, H. M.; Stevens, I. D. R. J. Chem. Soc. 1965, 1700.

(29) Amrich, M. J.; Bell, J. A. J. Am. Chem. Soc. 1964, 86, 292.

(30) Hoffmann, R. Tetrahedron 1966, 22, 539.

(31) Frey, H. M. Adv. Photochem. 1966, 225.

(32) Avila, M. J.; Figuera, J. M.; Menéndez, V.; Pérez, J. M.J. Chem. Soc., Faraday Trans. 1 1976, 72, 422.

(33) Bigot, B.; Ponec, R.; Sevin, A.; Devaquet, A. J. Am. Chem. Soc. 1978, 100, 6575.

(34) Mueller-Remmers, P. L.; Jug, K. J. Am. Chem. Soc. 1985, 107, 7275.

(35) Modarelli, D. A.; Platz, M. S. J. Am. Chem. Soc. 1993, 115, 470.

(36) Fedorov, I.; Koziol, L.; Mollner, A. K.; Krylov, A. I.; Reisler, H. J. Phys. Chem. 2009, 113, 7412.

(37) Mansoor, A. M.; Stevens, I. D. R. Tetrahedron Lett. 1966, 1733.

(38) Stevens, I. D. R.; Liu, M. T. H.; Soundararajan, N.; Paike, N. Tetrahedron Lett. 1989, 30, 481.

(39) Ford, F.; Yuzawa, T.; Platz, M. S.; Matzinger, S.; Fülscher, M. J. Am. Chem. Soc. 1998, 120, 4430.

(40) Seburg, R. A.; McMahon, R. J. J. Am. Chem. Soc. 1992, 114, 7183.

(41) Bernardi, F.; Olivucci, M.; Robb, M. A.; Vreven, T.; Soto, J. J. Org. Chem. 2000, 65, 7847.

(42) Jones, M. B.; Platz, M. S. J. Org. Chem. 1991, 56, 1694.

(43) Korneev, S. M. Eur. J. Org. Chem. 2011, 6153.

(44) Perez, J. M. J. Chem. Soc., Faraday Trans. 1 1982, 78, 3509.

(45) Bonneau, R.; Liu, M. T. H. J. Am. Chem. Soc. 1996, 118, 7229.

(46) Akasaka, T.; Liu, M. T. H.; Niino, Y.; Maeda, Y.; Wakahara, T.; Okamura, M.; Kobayashi, K.; Nagase, S. J. Am. Chem. Soc. 2000, $122,7134$.

(47) Arenas, J. F.; López-Tocón, I.; Otero, J. C.; Soto, J.J. Am. Chem. Soc. 2002, 124, 1728.

(48) Iacobucci, C.; Goetze, M.; Piotrowski, C.; Arlt, C.; Rehkamp, A.; Ihling, C. H.; Hage, C.; Sinz, A. Anal. Chem. 2018, 90, 2805.

(49) Procacci, B.; Roy, S. S.; Norcott, P.; Turner, N.; Duckett, S. B. J. Am. Chem. Soc. 2018, 140, 16855.

(50) O’Brien, J. G. K.; Jemas, A.; Asare-Okai, P. N.; am Ende, C. W.; Fox, J. M. Org. Lett. 2020, 22, 9415.

(51) Wang, J.; Burdzinski, G.; Gustafson, T. L.; Platz, M. S. J. Am. Chem. Soc. 2007, 129, 2597.

(52) Ziemianowicz, D. S.; Bomgarden, R.; Etienne, C.; Schriemer, D. C. J. Am. Soc. Mass Spectrom. 2017, 28, 2011.

(53) West, A.; Muncipinto, G.; Wu, H.-Y.; Huang, A.; Labenski, M. T.; Woo, C. ChemRxiv 2020, preprint DOI: 10.26434/chemrxiv.13373249.v1.

(54) Bunker, P. R.; Jensen, P.; Kraemer, W. P.; Beardsworth, R. J. Chem. Phys. 1986, 85, 3724.

(55) Irikura, K. K.; Goddard, W. A.; Beauchamp, J. L. J. Am. Chem. Soc. $1992,114,48$.

(56) Modarelli, D. A.; Platz, M. S. J. Am. Chem. Soc. 1991, 113, 8985.

(57) Gallo, M. M.; Schaefer, H. F. J. Phys. Chem. 1992, 96, 1515.

(58) Khodabandeh, S.; Carter, E. A. J. Phys. Chem. 1993, 97, 4360.

(59) Matzinger, S.; Fuelscher, M. P. J. Phys. Chem. 1995, 99, 10747.

(60) Admasu, A.; Gudmundsdóttir, A. D.; Platz, M. S.; Watt, D. S.; Kwiatkowski, S.; Crocker, P. J. J. Chem. Soc., Perkin Trans. 2 1998, 1093.

(61) Griller, D.; Liu, M. T. H.; Scaiano, J. C. J. Am. Chem. Soc. 1982, 104, 5549.

(62) Wang, L.; Tachrim, Z. P.; Kurokawa, N.; Ohashi, F.; Sakihama, Y.; Hashidoko, Y.; Hashimoto, M. Molecules 2017, 22, 1389. 
(63) Wang, L.; Ishida, A.; Hashidoko, Y.; Hashimoto, M. Angew. Chem. Int. Ed. 2017, 56, 870.

(64) Church, R. F. R.; Weiss, M. J. J. Org. Chem. 1970, 35, 2465.

(65) Dubinsky, L.; Jarosz, L. M.; Amara, N.; Krief, P.; Kravchenko, V. V.; Krom, B. P.; Meijler, M. M. Chem. Commun. 2009, 7378.

(66) Husain, S. S.; Forman, S. A.; Kloczewiak, M. A.; Addona, G. H.; Olsen, R. W.; Pratt, M. B.; Cohen, J. B.; Miller, K. W. J. Med. Chem. 1999, $42,3300$.

(67) Li, Z.; Hao, P.; Li, L.; Tan, C. Y. J.; Cheng, X.; Chen, G. Y. J.; Sze, S. K.; Shen, H.; Yao, S. Q. Angew. Chem. Int. Ed. 2013, 52, 8551.

(68) Walko, M.; Hewitt, E.; Radford, S. E.; Wilson, A. J. RSC Adv. 2019, 9, 7610 .

(69) Horne, J. E.; Walko, M.; Calabrese, A. N.; Levenstein, M. A.; Brockwell, D. J.; Kapur, N.; Wilson, A. J.; Radford, S. E. Angew. Chem. Int. Ed. 2018, 57, 16688.

(70) McCutcheon, D. C.; Lee, G.; Carlos, A.; Montgomery, J. E.; Moellering, R. E. J. Am. Chem. Soc. 2019, 142, 146.

(71) Ahad, A. M.; Jensen, S. M.; Jewett, J. C. Org. Lett. 2013, 15, 5060.

(72) Wu, B.; Jayakar, S. S.; Zhou, X.; Titterton, K.; Chiara, D. C.; Szabo, A. L.; Savechenkov, P. Y.; Kent, D. E.; Cohen, J. B.; Forman, S. A.; Miller, K. W.; Bruzik, K. S. Euro. J. Med. Chem. 2018, 136, 344.

(73) Al-Omari, M.; Banert, K.; Hagedorn, M. Angew. Chem. Int. Ed. 2005, 45, 309.

(74) Wagner, G.; Knoll, W.; Bobek, M. M.; Brecker, L.; van Herwijnen, H. W. G.; Brinker, U. H. Org. Lett. 2010, 12, 332.
(75) Cheng, W. W. L.; Chen, Z.-W.; Bracamontes, J. R.; Budelier, M. M.; Krishnan, K.; Shin, D. J.; Wang, C.; Jiang, X.; Covey, D. F.; Akk, G.; Evers, A. S. J. Biol. Chem. 2018, 293, 3013.

(76) Wallace, R. G. Aldrichimica Acta 1980, 3, 3.

(77) Flaxman, H. A.; Chang, C.-F.; Wu, H.-Y.; Nakamoto, C. H.; Woo, C. M. J. Am. Chem. Soc. 2019, 141, 11759.

(78) MacKinnon, A. L.; Taunton, J. Curr. Protoc. Chem. Biol. 2009, 1, 55.

(79) Wu, F.; Zhang, Y.; Sun, B.; McMahon, A. P.; Wang, Y. Cell Chem. Biol. 2017, 24, 252.

(80) Hall, T. M. T.; Porter, J. A.; Young, K. E.; Koonin, E. V.; Beachy, P. A.; Leahy, D. J. Cell 1997, 91, 85.

(81) Becke, A. D. Phys. Rev. A: At., Mol., Opt. Phys. 1988, 38, 3098.

(82) Becke, A. D. J. Chem. Phys. 1993, 98, 5648.

(83) Vosko, S. H.; Wilk, L.; Nusair, M. Can. J. Phys. 1980, 58, 1200.

(84) Stephens, P. J.; Devlin, F. J.; Chabalowski, C. F.; Frisch, M. J. J. Phys. Chem. 1994, 98, 11623.

(85) Frisch, M. J.; Pople, J. A.; Binkley, J. S. J. Chem. Phys. 1984, 80, 3265.

(86) Linden, A.; Vasella, A.; Witzig, C. Helv. Chim. Acta 1992, 75, 1572.

(87) Knoll, W.; Bobek, M. M.; Giester, G.; Brinker, U. H. Tetrahedron Lett. 2001, 42, 9161.

(88) Bobek, M. M.; Krois, D.; Brehmer, T. H.; Giester, G.; Wiberg, K. B.; Brinker, U. H. J. Org. Chem. 2003, 68, 2129.

(89) Dequirez, G.; Pons, V.; Dauban, P. Angew. Chem. Int. Ed. 2012, 51, 7384. 